\title{
Expression of mannose receptor and ligands for its cysteine-rich domain in venous sinuses of human spleen
}

\author{
Luisa Martinez-Pomares $^{1, *}$, Leif G Hanitsch ${ }^{1, \dagger}$, Richard Stillion ${ }^{1}$, Satish Keshav ${ }^{2}$ \\ and Siamon Gordon ${ }^{1}$ \\ ${ }^{1}$ Sir William Dunn School of Pathology, University of Oxford, Oxford, UK and ${ }^{2}$ Department of Medicine, \\ Royal Free and University College Medical School, London, UK
}

\begin{abstract}
The mannose receptor (MR) is a type I membrane molecule with two lectin activities. Mannose recognition takes place through the $\mathrm{C}$-type lectin-like carbohydrate recognition domains, while recognition of sulphated glycans is mediated by the cysteine-rich domain (CR). In murine spleen CR ligands are present in a subpopulation of macrophages $(\mathbf{M} \phi)$ placed in the marginal zone whereas MR-expressing cells consisting of $M \phi$ and nonvascular endothelia are located in the red pulp. No colocalisation of MR with CR ligands has been observed in murine tissues. In this manuscript we describe the distribution of MR and CR ligands in human spleen. In this organ we have detected a perfect colocalisation of MR with CR ligands in Lyve-1+ cells lining venous sinuses. These cells form a physical barrier for blood cells as they need to migrate through the sinuses in order to exit the splenic parenchyma and, in this way, contribute to the unique filtration function of this organ. Furthermore, unlike murine spleen, $\mathrm{CD} \mathrm{8}^{+}$red pulp M $\phi$ lack MR expression. Our results suggest an unexpected contribution of MR to splenic function through the recognition of sulphated ligands that could influence the filtering capability of this organ.
\end{abstract}

Laboratory Investigation (2005) 85, 1238-1249. doi:10.1038/labinvest.3700327; published online 1 August 2005

Keywords: mannose receptor; sulphated carbohydrates; human; rat; spleen

The mannose receptor (MR) is a type I membrane protein with three types of domain in the extracellular region; ${ }^{1-3}$ a cysteine-rich domain (CR), that is a member of the $\beta$-trefoil protein family, ${ }^{4}$ a domain containing fibronectin type II repeats and 8 C-typelectin-like domains (CTLD). The cytopasmic tail of the MR contains signals that mediate receptor internalisation and recycling., ${ }^{5,6}$ Two well-characterised lectin activities are mediated by the extracellular region of MR: CR binds sulphated sugars terminated in $\mathrm{SO}_{4}$-3-galactose (Gal), $\mathrm{SO}_{4}-4-\mathrm{N}$-acetylgalactosamine (GalNAc) and $\mathrm{SO}_{4}-3$-GalNAc; these modifications are present in glycoprotein hormones produced by the anterior pituitary, in chondroitin sulphate $\mathrm{A}$ and $\mathrm{B}$ and in sulphated Lewis ${ }^{\mathrm{a}}$ and Lewis ${ }^{\mathrm{x}} \cdot{ }^{7-9}$ The second lectin activity is mediated by

Correspondence: Dr L Martinez-Pomares, PhD, Sir William Dunn School of Pathology, South Parks Road, Oxford OX1 3RE, UK.

E-mail: luisa.martinez-pomares@path.ox.ac.uk

*Current address: School of Molecular Medical Sciences, Faculty of Medicine and Health Sciences, University of Nottingham Queen's Medical Centre, Nottingham NG7 2UH, UK

Current address: Engeldamm 28, Berlin D-10179, Germany

Received 23 February 2005; revised 15 June 2005; accepted 22 June 2005; published online 1 August 2005 several of its CTLD. ${ }^{10}$ CTLD4-7 recognise complex sugars terminated in mannose, fucose and $\mathrm{N}$-acetylglucosamine. ${ }^{5}$

Although MR is considered a macrophage $(\mathrm{M} \phi)$ marker its expression by other cell types has been well documented. ${ }^{11}$ In mice in addition to most tissue $\mathrm{M} \phi, \mathrm{MR}$ is present in hepatic and lymphatic endothelia and mesangial cells. In humans MR expression has been demonstrated in monocyte-derived $\mathrm{M} \phi$ and dendritic cells in vitro and in myeloid cells in dermis and tonsils and lymphatic endothelium in lymph nodes in situ. ${ }^{12-14}$

The spleen is a major filtration organ for microbes and blood cells and it is required to elicit responses against TI-2 antigens. It is divided into a lymphocyte-rich area (white pulp) and an erythrocyte-rich area (red pulp) where filtration and clearance take place, likely mediated by specialised $\mathrm{M} \phi$ populations. Splenic architecture varies among species. In rodents (mice and rats) a well-developed structure, the marginal zone ${ }^{15}$ has been described surrounding a clearly defined marginal sinus and containing specialised $\mathrm{M} \phi$ and B-cell populations. A more confusing picture emerges in the case of human 
spleen where the presence of a marginal sinus has been questioned; a marginal zone is solely defined on the basis of an accumulation of mature B cells and a perifollicular area has been described with increased numbers of red blood cells. ${ }^{16}$ Regarding the splenic red pulp, cords and sinuses have been clearly demonstrated in rats and humans. Splenic cords act as filtration beds receiving blood from terminal arterial vessels and conveying it to venous sinuses ${ }^{17}$ that contain, among others cells, mature $\mathrm{M} \phi$ involved in phagocytosis. The walls of the splenic sinuses consist of specialised endothelial cells with intercellular slits, that express the alpha chain of CD8 and thrombomodulin and are weakly positive for CD31. ${ }^{16}$ These cells are fixed to ring fibres of basement membrane-like material transversing the endothelial cells on their basal surface. Blood cells circulating through the red pulp flow across the wall of splenic sinuses between endothelial cells.

Analysis of MR and of CR ligands in mouse spleen showed a clear compartmentalisation with MR present in $\mathrm{M} \phi$ and nonvascular endothelia in the red pulp and CR ligands expressed by specialised $\mathrm{M} \phi$ surrounding B-cell follicles. ${ }^{11} \mathrm{MR}$ in red pulp is likely to be involved in the clearance of endogenous and microbe-associated mannosylated ligands. Cells bearing CR ligands migrate within the B-cell areas upon stimulation and then express CD11c, suggesting a role in antigen transport. ${ }^{18-20}$ CR ligands in spleen are exposed at the cell surface and mediate specific binding and internalisation of CR-bearing recombinant proteins introduced into the circulation. ${ }^{21}$ No association of $\mathrm{MR}^{+}$cells and cells expressing ligands for CR has been observed in this animal model ${ }^{11}$ and a soluble form of MR, identified in the human and mouse, has been proposed as the counter receptor for these CR ligands. ${ }^{22-25}$

In order to assess how the MR/CR ligand system may function across species, we have generated a chimaeric protein containing the human CR fused to mouse IgG2a $F_{c}$ to investigate the presence of CR ligands in human spleen and to compare their expression with that of the MR.

In this manuscript, we demonstrate that CR ligands (able to bind mouse and human CR) are present in equivalent areas in mouse and rat spleens suggesting that the role of CR ligands is likely to be conserved between these species. However, in human spleen CR ligands are present in venous sinuses in the red pulp. Remarkably and in contrast to the results obtained with rodent spleen, we observed a perfect colocalisation between MR and CR ligands and the absence of MR on $\mathrm{CD}^{+}{ }^{+}$red pulp $\mathrm{M} \phi$. The presence of $\mathrm{MR}$ and CR ligands in venous sinuses suggests a specific role for the MR/CR ligand interaction in human spleen in mediating adhesion of the venous sinuses cells and/or in controlling cell trafficking through the sinus.

\section{Materials and methods}

\section{Source of Tissues}

Rat tissues were obtained from animals bred at the Sir William Dunn School of Pathology.

Spleen tissue for immunohistochemistry was obtained from samples that were surplus to clinical requirements following tissue typing for solid organ transplantation. Unfixed $5 \mathrm{~mm}$ square blocks were frozen in OCT-embedding medium (Bayer Diagnostics, Berkshire, UK) and frozen slowly in a bath of dry ice and ethanol.

\section{Reagents}

Emr2-muFc was kindly provided by Dr Martin Stacey (University of Oxford) and muCR-huFc was prepared as described. ${ }^{20}$

\section{Generation of huCR-muFc}

The CR domain of the human MR was amplified using the primer $5^{\prime} \mathrm{Hu}$ CR: GGC ATC GGA TCC TGT CCA TCA GGA GAA GG and $3^{\prime}$ Hu CR: GCC ATC GCG GCC GCG CCT AGT AGC GTA TAC ATG GCT T using as template the plasmid pMMR265, kindly provided by Dr Maureen Taylor, University of Oxford. The PCR product contained a BamHI site at the $5^{\prime}$ end and a NotI site at the $3^{\prime}$ end. The hinge$\mathrm{CH} 2$ and $\mathrm{CH} 3$ regions of a non-Fc receptor-binding form of murine IgG2b was amplified using as template plasmid DNA generously provided by Lisa Gilliland (SWDSOP) using the primers $5^{\prime}$ muFc: GGC ATG GCG GCC GCA GAG CCC AGC GGG CCC ATT TCA AC and $3^{\prime} \mathrm{muFc}$ : GGC ATG TCT AGA TCA TTT ACC CGG AGA CCG GGA GAT. This PCR product had a NotI site at the $5^{\prime}$ end and an XbaI site at the $3^{\prime}$ end. The DNA fragments encoding huCR and $\mathrm{muFc}$ were cloned into the expression vector pcDNA.3 digested with BamHI and XbaI. This cloning strategy introduced three alanine residues between the huCR and $\mathrm{mFc}$. The resulting plasmid was transfected into 293T cells using genejuice (Novagen, Merk Biosciences Ltd, Nottingham, UK) and the recombinant huCR-muFc protein was purified from cell supernatants using protein A sepharose (Amersham Biosciences, UK).

\section{Sugar Binding Assay}

All washings and incubations were performed in $10 \mathrm{mM}$ Tris-HCl, $\mathrm{pH}$ 7.5, $10 \mathrm{mM} \mathrm{Ca}^{2+}, 0.154 \mathrm{M} \mathrm{NaCl}$ and $0.05 \%(\mathrm{w} / \mathrm{v})$ Tween 20.

Sugar polyacrylamide (PAA) substrates (Lectinity, Moscow) were coated onto the wells of ELISA plates (Nunc; Maxisorb) by incubation in $154 \mathrm{mM} \mathrm{NaCl}$ overnight at $37^{\circ} \mathrm{C}(50 \mu \mathrm{l} /$ well, sealed in a damp box $)$ at $5 \mu \mathrm{g} / \mathrm{ml}$. After coating, plates were washed five times. HuCR-muFc was incubated in the wells of 
coated plates $(50 \mu \mathrm{l} /$ well) for $2 \mathrm{~h}$ at room temperature. Binding was detected by incubation with antimouse IgG Fc-specific alkaline phosphatase-conjugated (Chemicon, Hampshire, UK). Plates were washed five times and developed with $p$-nitrophenyl phosphate substrate (Sigma-Aldrich, UK) in $100 \mathrm{mM}$ Tris-HCl, pH9.5, $100 \mathrm{mM} \mathrm{NaCl}, 5 \mathrm{mM}$ $\mathrm{MgCl}_{2}$. Absorbance was measured at $405 \mathrm{~nm}$. All assays were carried out in duplicate.

\section{Immunohistochemistry}

\section{Bright field}

Detection of sialoadhesin (Sn) and CR ligands in rat spleen: Fresh rat tissues were embedded in TissueTek OCT compound (Bayer Diagnostics) and were frozen on dry ice-cooled isopentane. Sections $(5 \mu \mathrm{m})$ were cut, air dried and stored at $-20^{\circ} \mathrm{C}$. Sections were thawed at room temperature, fixed in $2 \%$ paraformaldehyde in Hepes-buffered saline, permeabilized in $0.1 \%$ Triton X-100 in PBS, quenched using glucose oxidase, and blocked in 5\% normal sheep sera in PBS. Primary antibody (ED3 or isotype-matched control at $2 \mu \mathrm{g} / \mathrm{ml}$ from Serotec Ltd, Kidlington, UK) or murine Fc chimaeric proteins (huCR-muFc or Emr2-muFc) diluted in blocking buffer $(5 \mu \mathrm{g} / \mathrm{ml})$ were added and incubated for $1 \mathrm{~h}$ at room temperature. Binding was detected using peroxidase-conjugated sheep anti-mouse immunoglobulin Ig (Vector, Peterborough, UK). When the human Fc-derived protein muCR-huFc was used, blocking was performed using normal goat serum and binding was detected using peroxidaseconjugated goat anti-human IgG (Vector).

Detection of MR, CD68, Lyve-1 and CR ligands in human spleen: Fresh human spleens were embedded in Tissue-Tek OCT compound (Bayer Diagnostics) and were frozen as described above. Sections $(5 \mu \mathrm{m})$ were cut, air dried and frozen. Sections were thawed at room temperature, fixed in $2 \%$ paraformaldehyde in Hepes-buffered saline, permeabilized in 1\% Triton X-100 in PBS, quenched using glucose oxidase, and blocked with $5 \%$ normal sera in PBS. Sections were then incubated with primary mouse antibodies (15.2.2 (anti-MR) (HyCult biotechnololgy,) or anti-CD68 (Clone EBM11, Dakocytomation, Denmark), muFc-derived chimaeric proteins (huCR-muFc or Emr2-muFc) or polyclonal rabbit serum against human Lyve-1 (kindly provided by Dr David Jackson, University of Oxford) diluted in blocking buffer. In the case of mouse IgG and rabbit antiserum, incubation was performed at room temperature for $1 \mathrm{~h}$. Incubation with huCR-muFc and Emr2-muFc was performed at room temperature overnight. In all cases binding was detected using biotinylated horse anti-mouse IgG (Vector, Peterborough, UK) and the peroxidase-conjugated-ABC detection system (Vector). In some instances, huCRmuFc was used in the presence of $10 \mathrm{mM} \mathrm{SO}_{4}-4$ GalNAc (Dextra Laboratories, Reading, UK).

\section{Fluorescence}

Detection of CR ligands in human spleen was performed using a three-step amplification system by which huCR-muFc binding was detected using digoxigenin-conjugated anti-mouse (Chemicon) and FITC-conjugated anti-digoxigenin $\mathrm{Ab}$ (Roche Switzerland). For detection of MR, biotinylated 15.2.2 $\mathrm{mAb}$ and Cy5-conjugated streptavidin was used; for detection of CD68, nonconjugated antibody and Alexa 488-conjugated anti-mouse IgG was employed. To detect Lyve-1, polyclonal rabbit serum against human Lyve-1 and FITC-conjugated antirabbit IgG (Jackson ImmunoResearch Europe Ltd, Cambridgeshire, UK) were used. Sections were counterstained using DAPI and mounted using fluorescence-mounting media (Dakocytomation). Sections were analysed using a Zeiss Axioplan 2e microscope fitted with a 16 bit, $1024 \times 1024$ pixels CCD camera. Images were processed using Metamorph software. Adjustments were the same for all the images acquired for each experiment.

\section{Preparation of Tissue and Cell Lysates}

Human and rat spleen samples were cut using a sterile scalpel and placed in ice cold lysis buffer $(2 \%$ Triton X-100, $10 \mathrm{mM}$ Tris-HCl pH8, $150 \mathrm{mM} \mathrm{NaCl}$, $2 \mathrm{mM}$ EDTA, $10 \mathrm{mM} \mathrm{NaN}$ ) containing protease inhibitors (Roche, 1 tablet $/ 10 \mathrm{ml}$ lysis buffer). Tissue pieces were processed using a Dounce homogeniser and protein solubilisation was aided by further incubation at $4^{\circ} \mathrm{C}$ for $45 \mathrm{~min}$. Nonsolubilised material was removed by centrifugation at $4^{\circ} \mathrm{C}$ in a microfuge (2000 r.p.m. for $5 \mathrm{~min}$ followed by 13000 r.p.m. for $15 \mathrm{~min}$ ). Protein concentration was measured using the BCA method (Pierce, Perbio Science, Cheshire, UK). Lysates were aliquoted and kept frozen at $-80^{\circ} \mathrm{C}$. Cell lysates from human blood monocyte-derived $\mathrm{M} \phi$ cultured in six-well plates, were prepared by washing cells in ice cold PBS, addition of lysis buffer containing protease inhibitors and incubation at $4^{\circ} \mathrm{C}$ for $45 \mathrm{~min}$. Lysates were collected and cleared as described above. Culture supernatants were collected, centrifuged at 3000 r.p.m. $4^{\circ} \mathrm{C}$ for $10 \mathrm{~min}$ and stored at $-80^{\circ} \mathrm{C}$.

\section{Detection of MR in Human Spleen Lysates and in Cell Lysates and Supernatants from Human Monocyte-Derived M $\phi$ by Western Blot}

Protein lysates were electrophoresed in a 6 or $10 \%$ SDS-PAGE under nonreducing conditions and transferred to nitrocellulose filters (Amersham). Filters were blocked using $5 \%(\mathrm{w} / \mathrm{v})$ nonfat milk in PBS-T (PBS containing $0.01 \%(\mathrm{v} / \mathrm{v})$ Tween 20). Presence of MR was assessed by incubation with the anti-human MR mAb clone 15.2.2 (5 $\mu \mathrm{g} / \mathrm{ml}$ in blocking buffer) and species-absorbed horseradish peroxidase-conjugated anti-mouse IgG (Jackson ImmunoResearch). Binding was detected using the ECL reagent (Amersham Biosciences, UK). 


\section{Detection of CR Ligands in Human and Rat Spleen Lysates}

Protein lysates were electrophoresed and transferred to filters as before. CR ligands were detected using huCR-muFc or muCR-huFc $(10 \mu \mathrm{g} / \mathrm{ml}$ in blocking buffer) and species-absorbed horseradish peroxidase-conjugated anti-mouse IgG or anti-human IgG (Jackson ImmunoResearch) and visualised using the ECL system.

\section{Detection of Sn in Rat Spleen Lysates and in Preparations of CR Ligands}

Total protein lysates were electrophoresed and transferred to filters as before. Sn was detected using ED3 $\mathrm{mAb}(10 \mu \mathrm{g} / \mathrm{ml}$ in blocking buffer) and speciesabsorbed horseradish peroxidase-conjugated antimouse IgG (Jackson ImmunoResearch) and visualised using the ECL system. To select for CR ligands, huCR-muFc and muCR-huFc were crosslinked to protein A sepharose using dimethyl pimelimidate (Sigma) as coupling reagent as described. ${ }^{26}$ Rat spleen lysates $(1.5 \mathrm{ml})$ were precleared using protein A sepharose $(300 \mu \mathrm{l})$ for $2 \mathrm{~h}$ at $4{ }^{\circ} \mathrm{C}$. $300 \mu \mathrm{l}$ of precleared lysate was incubated with $30 \mu \mathrm{l}$ of huCR-muFc-sepharose or muCR-huFc-sepharose in the presence or absence of $10 \mathrm{mM} \mathrm{SO}_{4}-4-G a l N A c$, or with $30 \mu \mathrm{l}$ of protein A-sepharose for $4 \mathrm{~h}$ at $4^{\circ} \mathrm{C}$. After incubation beads were collected and washed three times in lysis buffer containing protease inhibitors. Bound material was released by boiling in SDSPAGE-loading buffer, electrophoresed and transferred to nitrocellulose as before. Sn was detected as described above but using ED3 at $5 \mu \mathrm{g} / \mathrm{ml}$.

\section{Detection of CR Ligands in Human Spleen Lysates Through Enrichment and Ligand Blot Analysis}

Human spleen lysate $(1 \mathrm{ml})$ was precleared using protein A sepharose $(300 \mu \mathrm{l})$ for $2 \mathrm{~h}$ at $4^{\circ} \mathrm{C}$. In all, $500 \mu \mathrm{l}$ of precleared lysate was incubated with $50 \mu \mathrm{l}$ of muCR-huFc-sepharose in the presence or absence of $10 \mathrm{mM} \mathrm{SO}-4-\mathrm{GalNAc}$ over night at $4{ }^{\circ} \mathrm{C}$. After incubation beads were collected and washed three times in lysis buffer containing protease inhibitors. Bound material was released by boiling in SDSPAGE-loading buffer, electrophoresed and transferred to nitrocellulose as before. CR ligands were detected as described above but using huCR-muFc at $10 \mu \mathrm{g} / \mathrm{ml}$.

\section{Results}

HuCR Binds $\mathrm{SO}_{4}$-3-Galactose, $\mathrm{SO}_{4}-3-\mathrm{N}$-AcetylGalactosamine and GalNAc In Vitro

To confirm that the CR from human MR has binding properties similar to those of its murine counterpart, binding of the chimaeric protein huCR-muFc (Figure 1a) to PAA-derived glycoconjugates containing $\mathrm{SO}_{4}-3-\mathrm{Gal}, \mathrm{SO}_{4}-3-\mathrm{GalNAC}$ and $\mathrm{SO}_{4}-4-\mathrm{GalNAc}$ or $\alpha-1-3-G a l N A c$ was assayed as described in Materials and methods. As shown in Figure 1b human CR displayed binding properties similar to those described for its murine counterpart and preferentially recognised galactose and $N$-acetyl-galactosamine sulphated in position 3 in a dose-dependent manner. No binding to nonsulphated ligands was observed.

\section{Detection of CR Ligands in Rat Spleen: huCR-muFc Has the Same Binding Properties as muCR-huFc In Situ}

As a means to assess the conservation among rodents of the selective expression of CR ligands in the marginal zone, and to compare the ability of huCR and muCR to recognise sulphated sugars in tissue sections, rat spleens were incubated with huCR-muFc and muCR-huFc as described in

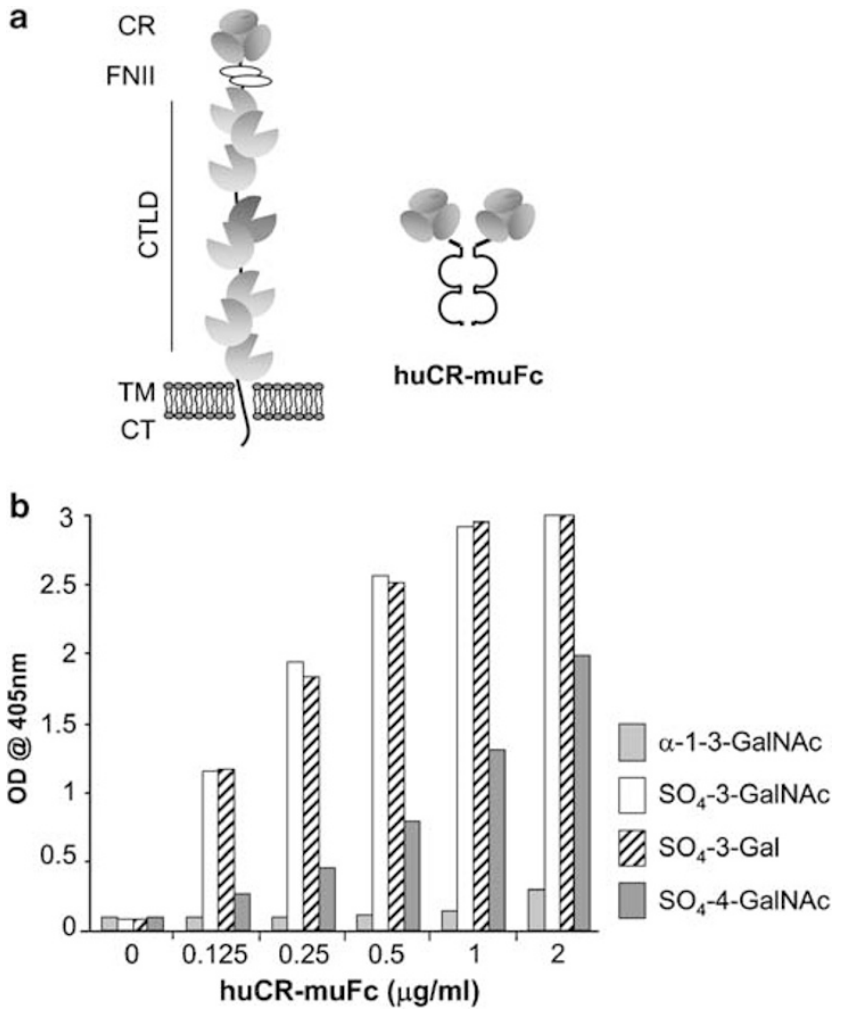

Figure 1 huCR-muFc recognises $\mathrm{SO}_{4}-3-\mathrm{Gal}, \mathrm{SO}_{4}-3-\mathrm{GalNAc}$ and $\mathrm{SO}_{4}$-4-GalNAc in vitro. (a) Schematic representation of human MR and the Fc chimaeric protein huCR-muFc. CR: cysteine-rich domain, FNII: domain-containing fibronectin type II repeats, CTLD: C-type carbohydrate recognition domain-like domain, TM: transmembrane domain, CT: cytoplasmic tail. (b) HuCR-muFc specifically binds the sulphated glycoconjugates $\mathrm{SO}_{4}-3-\mathrm{Gal}-\mathrm{PAA}$, $\mathrm{SO}_{4}-3-$ GalNAc-PAA and $\mathrm{SO}_{4}-4$-GalNAc-PAA in a dose-dependent manner. Enhanced recognition of glycoconjugates bearing $\mathrm{SO}_{4}$ at position 3 was observed as described previously for muCR-huFc and sMR. 
Materials and methods. As shown in Figure 2, both proteins labelled populations of cells placed in the marginal zone. These cells correspond to marginal zone $\mathrm{M} \phi$ and marginal zone metallophilic $\mathrm{M} \phi$, respectively, as they express $\mathrm{Sn}$ shown by the labelling of serial sections with the anti-Sn $\mathrm{mAb}$ ED3. ${ }^{16}$ Unlike in mice, CR ligands in rat spleen extend to the outer and inner marginal zone and do not show the restricted expression to areas of the marginal zone associated with B-cell follicles. Restricted expression of CR ligands in cells observed in the white pulp could correspond to migrated myeloid cells. No specific labelling was observed with the control proteins Emr2-muFc and human IgG1, or with the secondary detection reagent alone. Specific recognition through the lectin region of huCR was confirmed by the complete inhibition observed in the presence of soluble $\mathrm{SO}_{4}$-4-GalNAc.

\section{Detection of huCR and muCR Ligands in Rat Spleen by Ligand Blot}

In mice several CR ligands can be detected in protein lysates after electrophoresis and transfer to filters. These ligands include species with rMW ranging from 240 to $100 \mathrm{kDa}$ two of which have been characterised as Sn and low MW isoforms of CD45. ${ }^{26}$ To investigate the nature of the ligands recognised by huCR and muCR in rat spleen, splenic protein lysates were electrophoresed, transferred to nitrocellulose and incubated with huCR-muFc and muCR-huFc as described in Materials and methods. In agreement with the identical distribution of huCR and muCR ligands observed in spleen sections, huCR-muFc and muCR-huFc recognised the same ligands in rat spleen lysates by ligand blot analysis. A major band that comigrated with rat Sn (detected using mAb ED3) was observed (Figure 3a). To

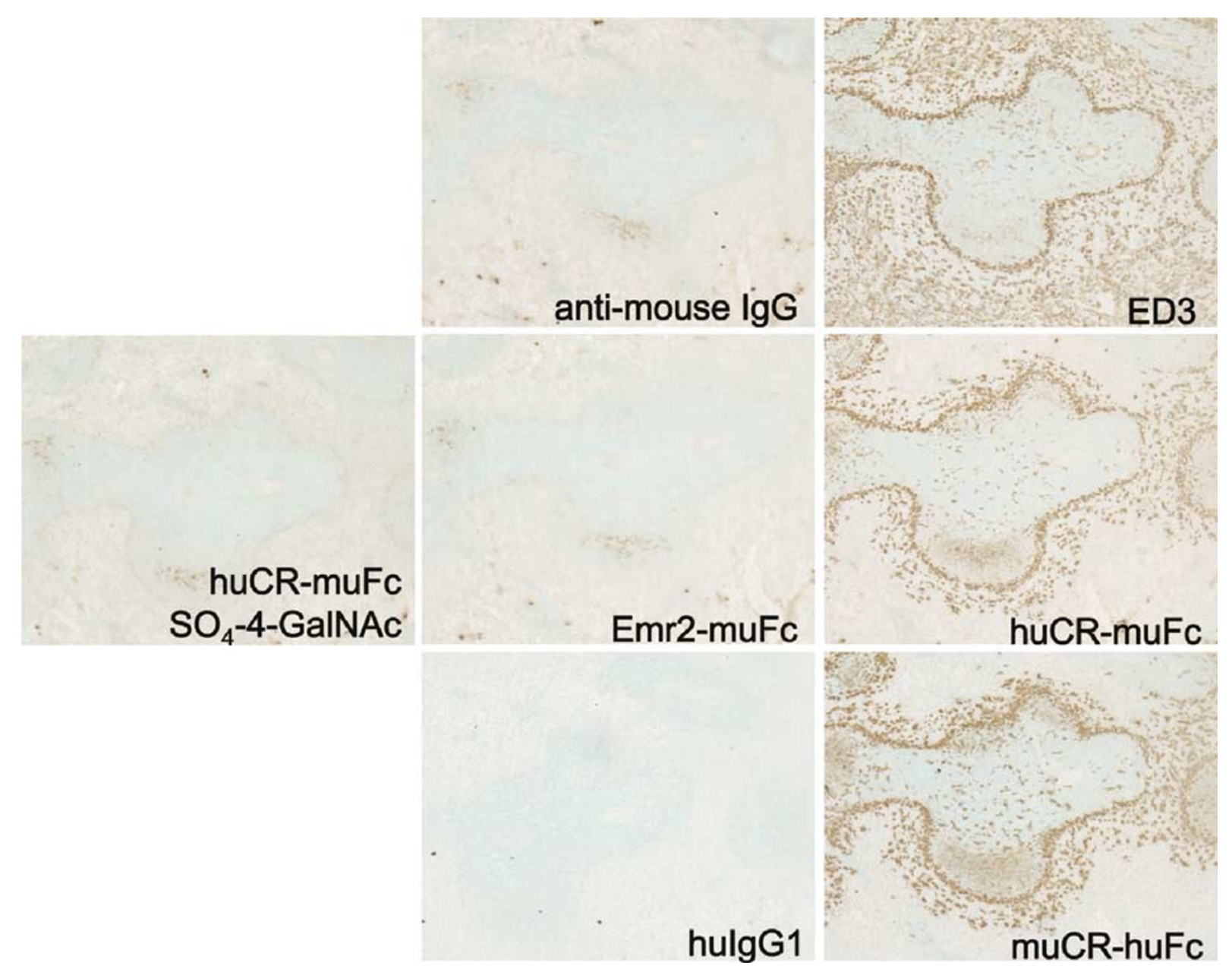

Figure 2 CR ligands can be detected using muCR-huFc and huCR-muFc in the marginal zone of rat spleen. Rat spleen sections were incubated with the chimaeric proteins huCR-muFc, muCR-huFc and the anti-rat Sn-specific mAb ED3 as described in Materials and methods. Human IgG1, Emr2-muFc and mouse IgG1 (isotype control for ED3, data not shown) were used as negative controls. HuCRmuFc binding was inhibited in the presence of $\mathrm{SO}_{4}-4$-GalNAc. Identical specific labelling of cells located in the outer and inner marginal zone can be observed with huCR-muFc and muCR-huFc. These cells correspond to marginal zone and marginal zone metallophilic M $\phi$ based on the expression of sialoadhesin. Additional cells bearing CR ligands can also be observed in the white pulp. These could correspond to migrating cells as described in the mouse. Magnification $\times 10$. 
confirm that rat splenic Sn was a counter-receptor for huCR and muCR, huCR and muCR ligands in rat spleen lysates were selected as described in Materials and methods and the presence of Sn in these preparations was tested by Western blot. Dependence for sulphated sugar recognition was determined by performing the selection in the presence or absence of soluble $\mathrm{SO}_{4}-4-G a l N A c$. As shown in Figure $3 \mathrm{~b}$ two bands immunoreactive with the antiSn mAb could be detected in the absence of inhibitor. The lower band (labelled with an arrow) colocalised with the band recognised by this mAb in total protein lysates. The upper band (labelled with an arrowhead) could correspond to a protein complex containing Sn. This was also observed in
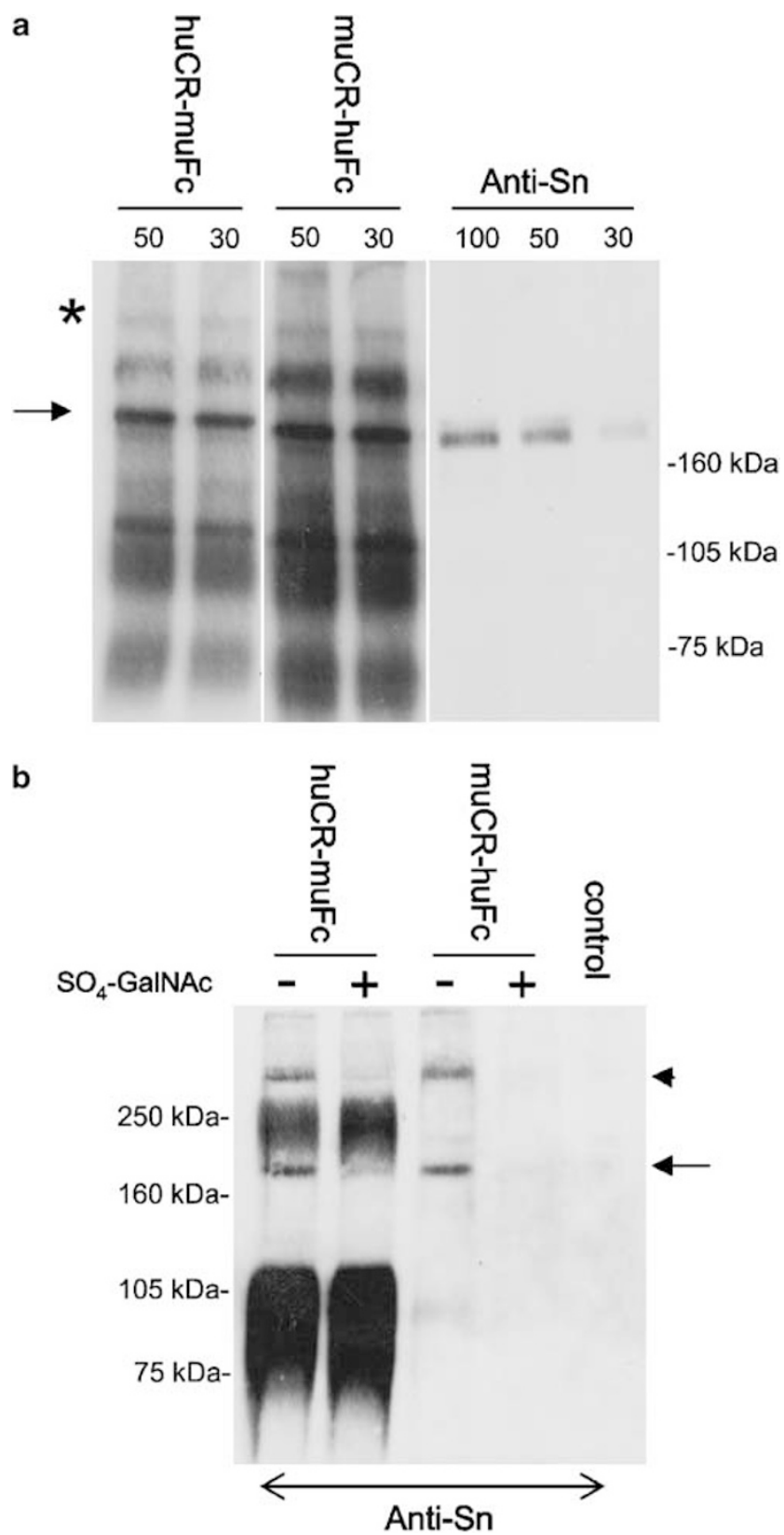

the case of mouse Sn selected based on its ability to bind muCR. ${ }^{26}$ A band with a similar rMW was observed in the ligand blots presented in Figure 3a (labelled with a star). These results reinforce the similarity between the mouse and rat system and demonstrate that the detection of CR ligands by ligand blot using huCR-muFc is feasible.

\section{HuCR-Ligands can be Detected in the Red Pulp of Human Spleen}

The marginal zone of human spleen lacks the classical marginal zone-associated $\mathrm{M} \phi$ characterised in rodents and it was of interest to investigate which cell population in this organ, if any, would display the sulphated ligands recognised by MR. Once we had determined that the huCR-muFc protein provided a suitable probe for in situ studies, we investigated its binding to normal human spleen. As shown in Figure 4a, a distinct pattern could be observed in which sinuses occupying the entire red pulp were recognised by huCR-muFc. Specificity was confirmed by the lack of labelling observed with Emr2-muFc (data not shown) and the inhibition of huCR-muFc binding in the presence of $\mathrm{SO}_{4}-4$ GalNAc. Though CR ligands could be consistently found in three independent spleen samples, their detection was favoured by tissue permeabilisation and overnight incubation with huCR-muFc. We were unable to detect CR ligands in human spleen lysates by ligand blot analysis under conditions in which the ligands present in rat spleen were readily detectable (Figure 5a). To increase the level of detection we selected CR ligands from human spleen lysates using muCR-huFc coupled to protein $A$ and probed them with huCR-muFc (Figure 5b). This analysis revealed the presence of three putative CR ligands in human spleen lysates that were absent when the selection was performed in the presence of

Figure 3 Analysis of CR ligands in rat spleen lysates. (a) HuCR and muCR recognise the same ligands in rat spleen lysates. Different amounts of protein lysates from rat spleen were transferred to nitrocellulose and incubated with huCR-muFc or muCR-huFc (50 and $30 \mu \mathrm{g}$ ) or with anti-Sn mAb (100, 50 and $30 \mu \mathrm{g}$ ) as described in Materials and methods. HuCR-muFc and muCR-huFc recognised a major band situated between the 160 and $250 \mathrm{kDa}$ MW markers (see arrow) that could correspond to Sn as it colocalises with the band recognised by the anti-Sn mAb. Other minor species with rMW above $50 \mathrm{kDa}$ were also detected. No binding of the secondary reagent used for detection was observed (data not shown). (b) Sn is present in preparations of huCR and muCR ligands. HuCR and muCR ligands were enriched in the presence $(+)$ or absence $(-)$ of $\mathrm{SO}_{4}-4$-GalNAc as described in Materials and methods. Selected proteins were analysed by Western blot using anti-Sn mAb. Two bands immunoreactive with this mAb (marked with arrow and arrowhead) were detected in the absence of inhibitor. Additional bands present in the preparation of huCR ligands in the presence and absence of inhibitor could correspond to huCR-muFc eluted from the sepharose as they were recognised by the anti-mouse IgG reagent used for detection (data not shown). Control line contained proteins selected using protein A sepharose. 
a

\section{huCR-muFc $+\mathrm{SO}_{4}-4-\mathrm{GalNAc}(10 \mathrm{X})$}

b

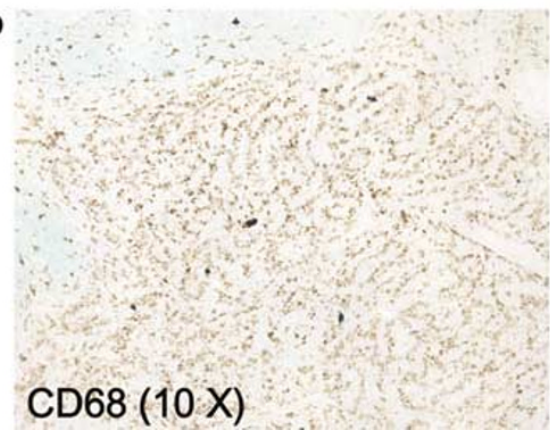

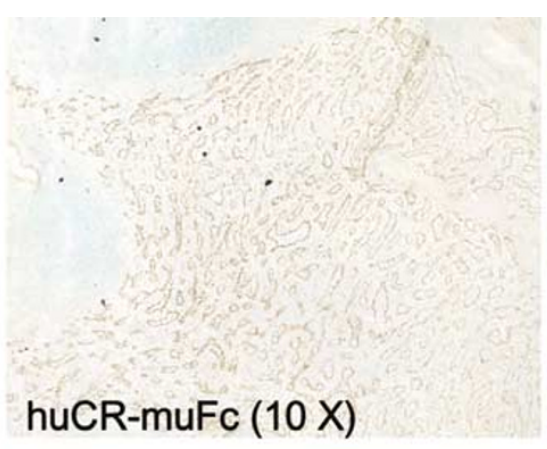

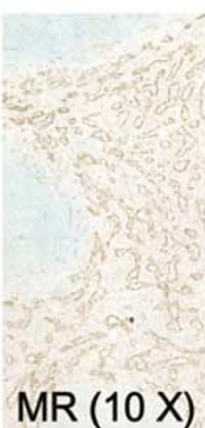

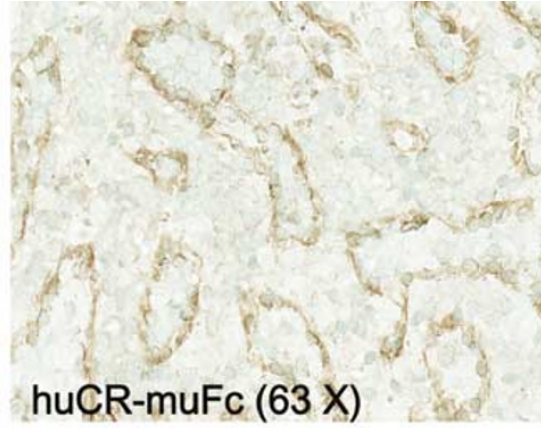

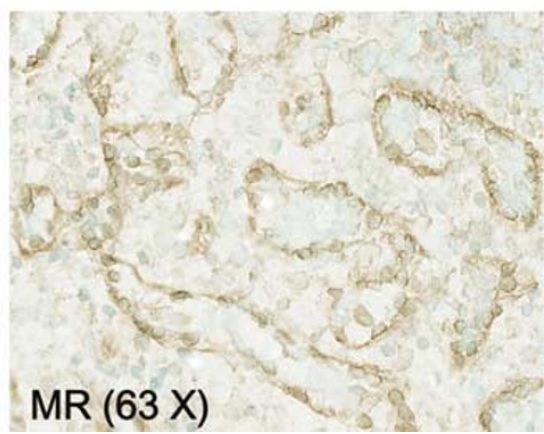

Figure 4 Analysis of CR ligands and MR distribution in human spleen by immunohistochemistry. (a) Detection of CR ligands in normal human spleen. Human spleens were incubated with $5 \mu \mathrm{g} / \mathrm{ml}$ of huCR-muFc and Emr2-muFc (data not shown) in the presence or absence of $\mathrm{SO}_{4}$-4-GalNAc and binding was detected as described above. Specific labelling was observed in the red pulp of the spleen of structures resembling venous sinuses. Similar results were obtained using 2.5 and $10 \mu \mathrm{g} / \mathrm{ml}$ (data not shown). (b) Analysis of MR distribution in normal human spleen. Distribution of MR in human spleen was analysed using the anti-MR mAb 15.2.2. MR expression was largely confined to the red pulp to structures similar to those containing CR ligands. No similarities with the distribution of the M $\phi$-restricted marker CD68 were observed.

$\mathrm{SO}_{4}$-4-GalNAc. Two of these displayed a very high rMW which would suggest that the major counter-receptors for CR in human spleen are large molecules or form SDS-resistant complexes. The third CR ligand migrated just below the $75 \mathrm{kDa} \mathrm{MW}$ marker.

\section{MR is Absent in CD68 ${ }^{+} \mathrm{M} \phi$ in Human Spleen, but is Highly Expressed by Lyve-1 ${ }^{+}$Cells Lining Venous Sinuses where it Colocalises with CR Ligands}

In order to investigate the functional relationship between MR and CR ligands in human spleen, we investigated MR distribution in this organ and compared it to that of CD68 (macrosialin), a lysosomal-associated glycoprotein abundant in $\mathrm{M} \phi$. When the expression of both markers was compared in serial sections it was clear that both receptors were present in completely different cell populations. Furthermore, MR distribution closely resembled the pattern observed for CR ligands as it was found in cells lining venous sinuses in the red pulp (Figure 4b). These results were confirmed by double immunofluorescence as no colocalisation was observed between MR and CD68 in the splenic red pulp parenchyma. Detailed analysis of the sections showed the presence of very few $\mathrm{MR}^{+} \mathrm{CD}{ }^{+}$cells with typical $\mathrm{M} \phi$ morphology. In contrast to the string-like labelling observed in the sinus-lining cells, MR in these $\mathrm{M} \phi$ had a distinct endosomal distribution in agreement with its role as an endocytic receptor. In contrast to CD68, a perfect colocalisation was observed between the lymphatic endothelial marker Lyve- $1^{27}$ and MR supporting the characterisation of these $\mathrm{MR}^{+}$cells as sinus-lining cells (Figure 6). Fluorescence labelling was also employed to determine if the resemblance between MR and CR ligand-distribution observed in bright field was due to colocalisation. Results shown in Figure 7 demonstrate that CR ligands and MR colocalise in red pulp venous sinuses, in the area of the cell facing the splenic parenchyma suggesting a role for this interaction in cell adhesion. It is noteworthy that the $\mathrm{MR}^{+} \mathrm{CD}^{+}{ }^{+}$cells observed within the sinuses do not contain CR ligands (Figure 6). No expression of collagen I or III was found associated with MR (data not shown).

\section{MR in Human Resembles the Cell-Associated form of MR Expressed by Human Monocyte-Derived M $\phi$}

In the mouse, a soluble form of MR (sMR) has been suggested as the counter-ligand for the CR ligands observed in the marginal zone as it is thought that under some circumstances, sMR present in serum could be targeted to these cells. To investigate if the 
a

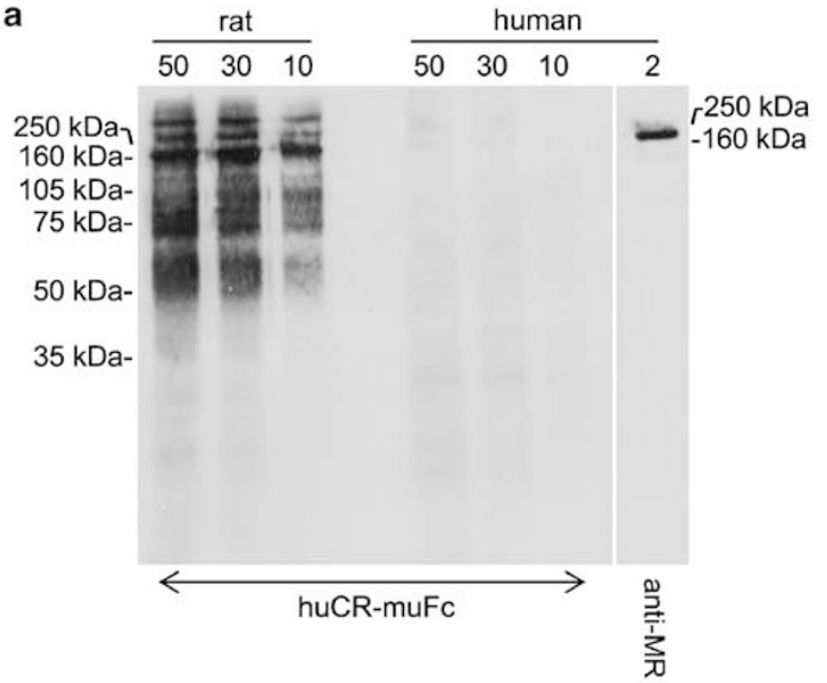

b

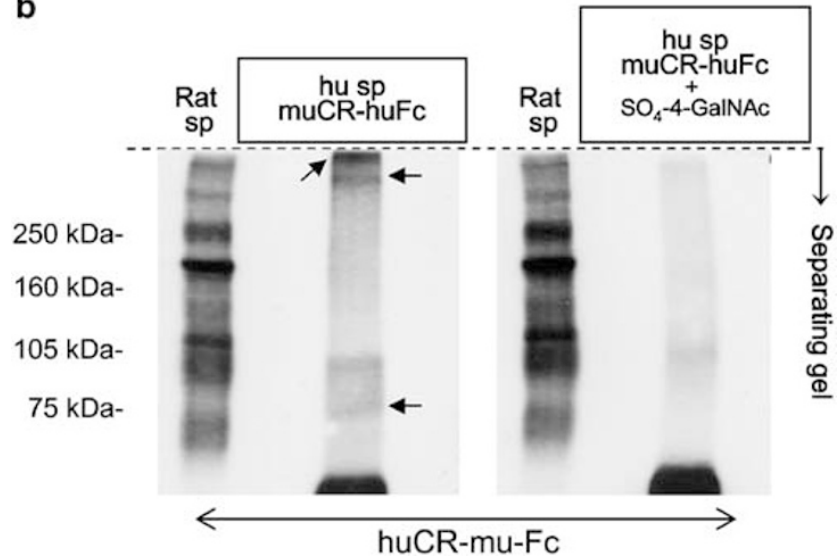

Figure 5 Characterisation of CR ligands in human spleen. (a) HuCR ligands in human spleen were not detected by ligand blot analysis. Different amounts $(10,20$ and $50 \mu \mathrm{g}$ ) of protein lysates from human and rat spleen were transferred to nitrocellulose and incubated with huCR-muFc as described. Specific binding of huCR-muFc to rat, but not human spleen lysates was observed. Integrity of the protein preparation was demonstrated by the detection of full-size MR in $2 \mu \mathrm{g}$ of lysate (anti-MR). No additional ligands were found at the lower MW range in the rat lysate. (b) Detection of huCR ligands in preparation of human spleen enriched for CR ligands. MuCR ligands in human spleen lysate were selected in the presence or absence of $\mathrm{SO}_{4}-4$-GalNAc as described in Materials and methods. MuCR-huFc was used because of the high background obtained when using huCRmuFc-sepharose, probably due to inefficient crosslinking of the mouse $\mathrm{FC}_{\mathrm{C}}$ to protein A. Enriched products were analysed by ligand blot using huCR-muFc. Three putative CR ligands (labelled with arrows) were observed in the absence of competitor.

colocalisation of MR with CR ligands could be due to the trapping of sMR in the sinuses, comparison of the rMW of human splenic MR with that of cMR and sMR produced in human $\mathrm{M} \phi$ cultures was performed by Western blot analysis. As shown in Figure 8, no sMR could be detected in splenic lysates even at high protein concentrations suggesting that the bulk of MR observed in human spleen corresponds to cell-associated receptor.

\section{Discussion}

In this manuscript, we describe the distribution of the MR and its CR ligands in human spleen. The results we have obtained can be summarised as follows: (i) MR is widely expressed in the red pulp of human spleen but is not present in red pulp $\mathrm{CD}^{+} 8^{+} \mathrm{M} \phi$. It is located in cells lining venous sinuses that express the nonvascular endothelial marker Lyve-1; (ii) MR is absent from the human splenic white pulp suggesting lack of expression by splenic dendritic cells at least under normal conditions; (iii) the CR of human $\mathrm{MR}$ recognises $\mathrm{SO}_{4}-3$ Gal, $\mathrm{SO}_{4}-3-\mathrm{GalNAc}$ and $\mathrm{SO}_{4}-4-\mathrm{GalNAc}$ in vitro and marginal zone-associated ligands in rat spleen in situ; (iv) CR ligands colocalise with MR in the venous sinuses.

We consider it highly likely that the ligands recognised by the CR domain in rat and human spleen correspond to sulphated carbohydrates based on the following observations: (a) Under no circumstances has a nonsulphated ligand been described for CR. $^{7}$ (b) Crystallographic studies have determined the residues in muCR required for sulphated carbohydrate binding. ${ }^{4}$ Sequence analysis demonstrated that all these residues are conserved in huCR. (c) The interaction between muCR and sulphated sugars takes place through a neutral binding pocket where soluble monomeric $\mathrm{SO}_{4}-4$ GalNAc would bind. Therefore, it is unlikely that this reagent would block hypothetical additional binding sites through steric hindrance. (d) CR does not undergo conformational changes upon interaction with sulphated carbohydrates. ${ }^{4}$ Therefore, it is unlikely that a second binding site could have been eliminated through conformational changes induced by our inhibitor. (e) Our results indicate that muCR and huCR have the same binding specificity: (e.1) the sugar-binding assay shown in Figure 1, is consistent with data published for muCR, (e.2) the distribution of muCR-huFc and huCR-muFc ligands in rat spleen sections is identical (Figure 2), (e.3) muCR-huFc and huCR-muFc recognised the same bands in rat spleen protein lysates by ligand blot analysis (Figure 3a) and (e.4) muCR-huFC and huCR-muFc shared the ability to interact with rat $\mathrm{Sn}$ in a sulphate-dependent manner (Figure $3 b$ ).

\section{The Marginal Zone in Rodents and Humans}

Using rat spleen as a model to validate the probe used to search for huCR ligands we have further confirmed the differences observed between mice and rats regarding the phenotype of marginal zoneassociated $\mathrm{M} \phi .^{28,29}$ In mice only marginal zone metallophilic $\mathrm{M} \phi$ express high levels of Sn and CR ligands $s^{11,20}$ while in rats the expression of both markers extends to the $\mathrm{M} \phi$ placed at the outer marginal zone. Further work is required to test if CR ligands are also exposed to the extracellular milieu 

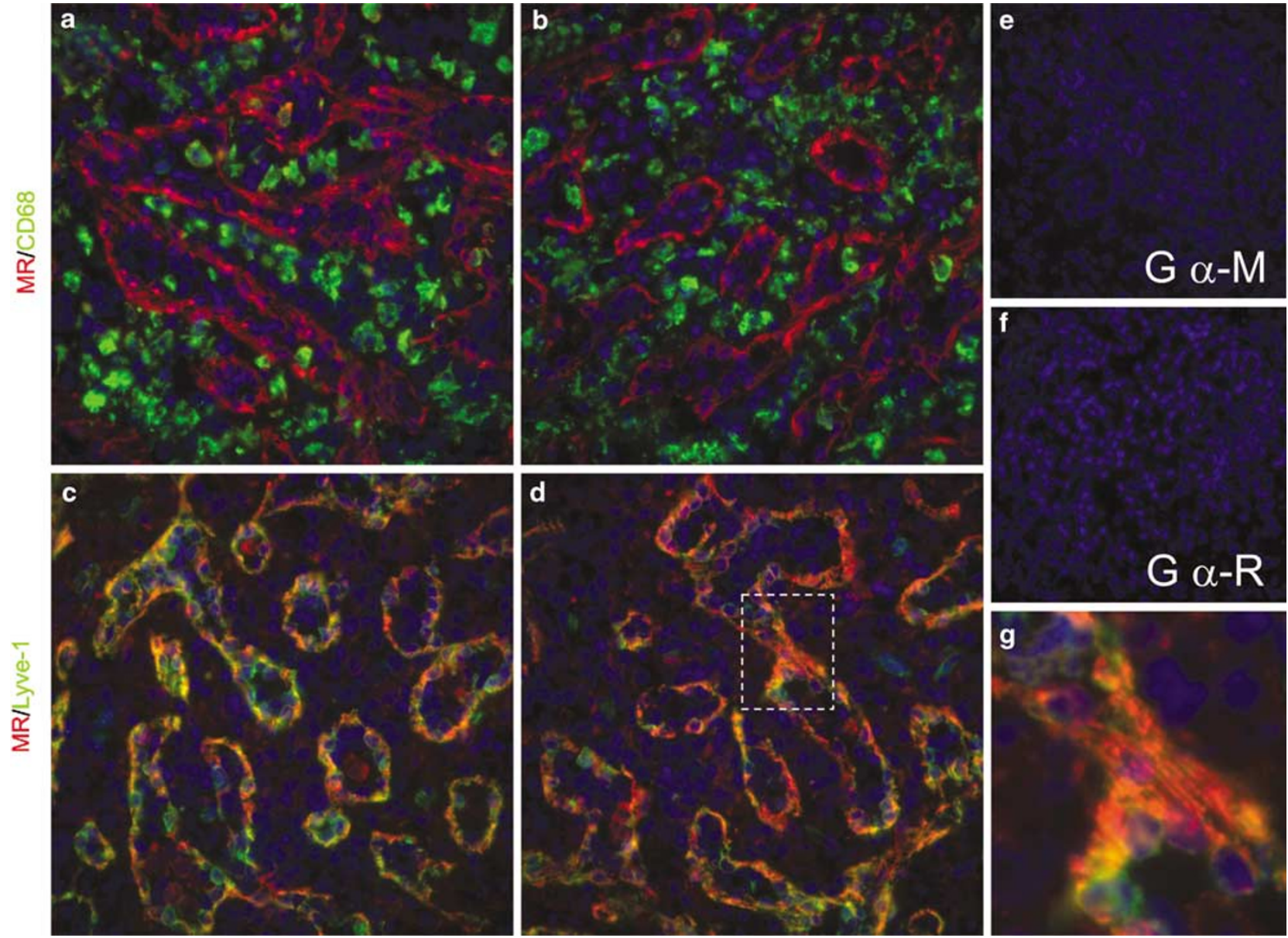

Figure 6 MR is present in Lyve-1+ ${ }^{+}$cells lining venous sinuses in human spleen. MR-specific fluorescent labelling in human spleen (in red) does not colocalise with CD68-specific labelling (in green, (a) and (b)) but colocalises with Lyve-1-specific labelling (in green, (c) and (d)). (e) and (f) show labelling obtained with anti-mouse IgG ( $\mathrm{G} \alpha-\mathrm{M})$ and anti-rabbit IgG (G $\alpha$-R) secondary antibodies. Inset (g) shows detail of (d) in which putative ring fibres double labelled with MR and Lyve-1 can be observed.

in rats ${ }^{21}$ and how their expression relates to MR distribution. We have been unable to detect CR ligands in any area surrounding the white pulp in human spleen. Even though Sn expression has been described in $\mathrm{M} \phi$ located in the perifollicular zone, ${ }^{16}$ our results suggest that in these cells it lacks the post-translational modification recognised by human CR.

\section{The Role of MR and CR Ligands in Venous Sinuses}

MR is present in most $\mathrm{M} \phi$ populations and in liver sinusoidal cells of the mouse where it is predicted to mediate clearance of endogenous and pathogenassociated molecules. MR expression has also been observed in nonvascular endothelia in mouse lymph nodes and spleen. ${ }^{11}$ Little information is available regarding the role of MR in these endothelial-like cells. In human lymph nodes a role has been proposed for MR expressed by lymphatic endothelia in cell adhesion through an interaction with Lselectin on $\mathrm{T}$ cells. ${ }^{30}$ Further work is required to understand how this receptor, mostly located in the endosomal compartment in $\mathrm{M} \phi$ and cultured cells (NIH3T3 and CHO cells), could mediate selective cell adhesion. These studies should include the analysis of the cellular biology of MR in endotheliallike cells. A role for MR in cell adhesion is further supported by its newly recognised ability to bind collagens through its FNII domain (MartinezPomares et al, manuscript in preparation). In this regard, it is noteworthy that no colocalisation of MR and collagens I and III has been observed in human spleen (data not shown).

Preliminary analysis of the cellular distribution of MR within venous sinus-lining cells indicated a fibrillar distribution that in some cases was associated with structures that resemble ring fibres where Lyve-1 was also present (see panel g in Figure 6 ). We consider two possible explanations for the presence of CR ligands colocalising with MR in venous sinuses. First, that the sulphated carbohydrates recognised by $\mathrm{CR}$, such as chondrotin sulphate, ${ }^{7}$ are located in the specialised basal membrane to which the endothelial cells attach. 


\section{huCR-muFc/MR}

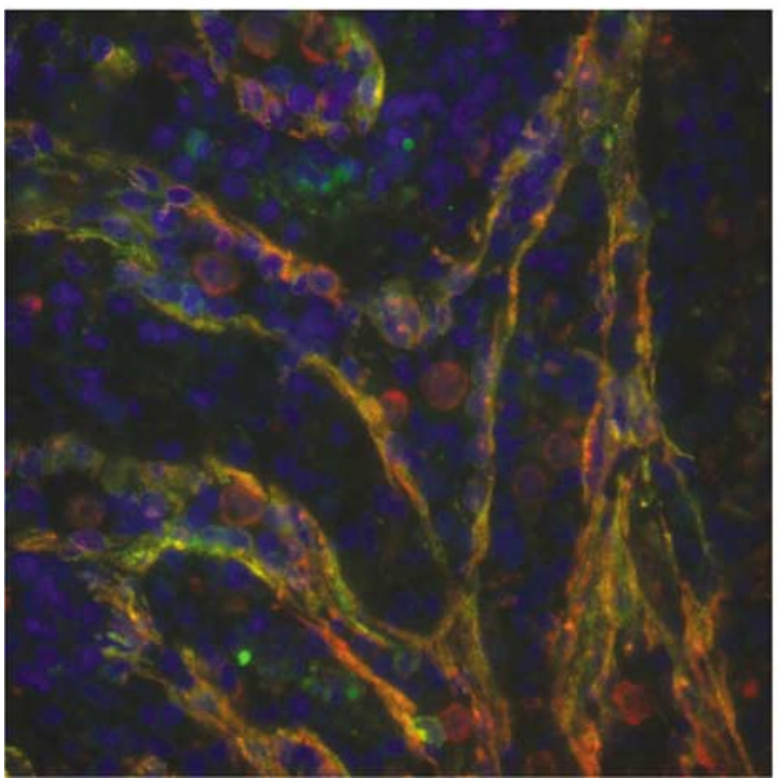

huCR-muFc $+\mathrm{SO}_{4}-4-\mathrm{GalNAc} / \mathrm{MR}$

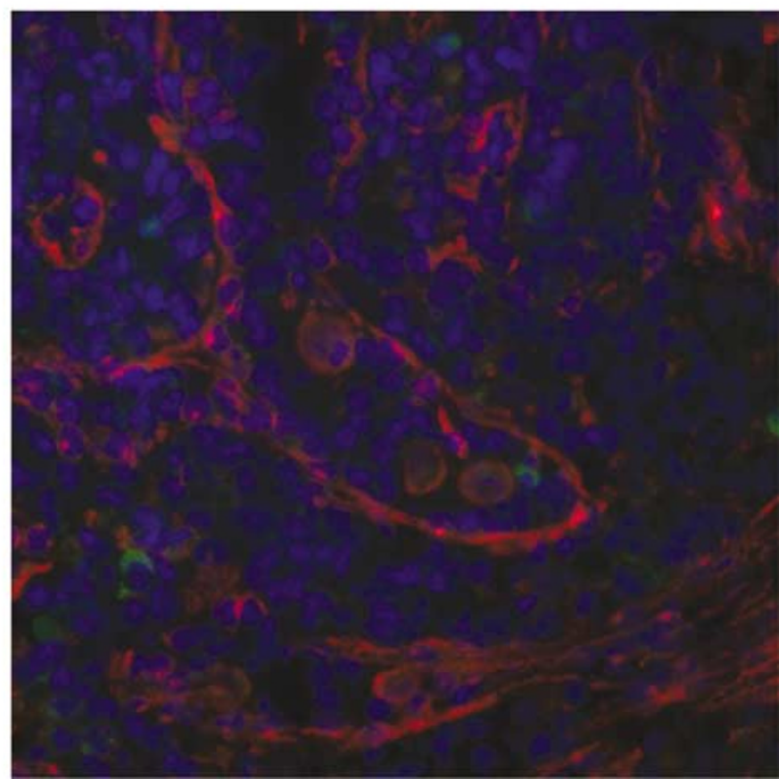

Figure 7 MR- and CR ligands colocalise in venous sinuses of splenic red pulp. Fluorescent labelling of CR ligands (in green) and MR (in red) in the presence or absence of $\mathrm{SO}_{4}-4-G a l N A c$ demonstrates that there is a perfect colocalisation of the MR and ligands for the CR in venous sinuses.

$\frac{\text { Spleen }}{12 \quad 6} \quad \frac{M \phi}{\mathrm{C} \quad \mathrm{S}}$

$250 \mathrm{kDa}-$

$160 \mathrm{kDa}-$

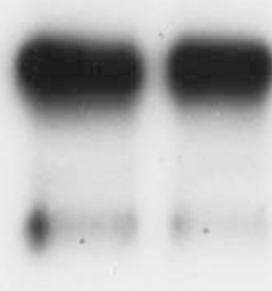

$105 \mathrm{kDa}$

Figure 8 Splenic MR has the same rMW as cMR produced by human $\mathrm{M} \phi$. Protein lysates (12 and $6 \mu \mathrm{g}$ ) of human spleen were electrophoresed alongside lysate $(14 \mu \mathrm{g})$ and supernatants ( $45 \mu \mathrm{l}$ of $9 \mathrm{~cm}^{2}$ dish containing $2 \mathrm{ml}$ media with $2 \%$ autologous human serum) of cultured human monocyte-derived $\mathrm{M} \phi$ and transferred to a nitrocellulose filter. Cell-associated and soluble MR were detected in $\mathrm{M} \phi$ cell lysate and supernatant, respectively. Splenic MR comigrated with the cell-associated form of MR present in $\mathrm{M} \phi$.

The uniqueness of this structure is exemplified by the restricted expression of collagen XVII in the ring fibres together with the integrin subunit $\alpha 6$. $^{31}$ This pattern of expression suggests the formation of a firm hemidesmosome-like contact site between the intermediate filaments of the sinusoidal endothelial cells and the ring fibre matrix. Second, the presence of these CR ligands could be due to MR-mediated uptake of sulphated ligands from the circulation. Detection of CR ligands was consistent but low probably due to low abundance or limited accessibility as the sulphated sugars recognised by our probe could already be interacting with the CR domain of the endogenous cell-associated MR. The ligand blot analysis shown in Figure 5b suggests that MR itself is not a CR ligand as no ligand with a rMW of $175 \mathrm{kDa}$ was detected by our analysis. Intriguingly, a minor band with an approximate rMW of $70 \mathrm{kDa}$ was observed. This mobility is similar to that displayed by glycosylated Lyve-1 in SDS-PAGE and raises the possibility of an interaction between MR and Lyve-1 in human spleen.

\section{Absence of MR in Human Red Pulp M $\phi$}

In humans, MR expression in myeloid cells is supported by its detection in cultured $\mathrm{M} \phi$ and dendritic cells in vitro and in myeloid populations in dermis and tonsils. Therefore, it was unexpected to find that red pulp $\mathrm{CD} 68^{+} \mathrm{M} \phi$ in human spleen did not express this receptor.

Mannosylation has been used as a means to deliver glucocerebrosidase to putative $\mathrm{M} \phi$ in Gaucher patients. ${ }^{32}$ Our results indicate that, at least in normal spleen, it is unlikely that MR mediates enzyme uptake by red pulp $\mathrm{M} \phi$. These observations are in agreement with the low MR expression observed in Gaucher's cells, ${ }^{33}$ which suggest that other mannose receptors and/or cells could be 
involved in the internalisation of mannosylated glucocerebrosidase.

In summary, our results provide evidence for an interaction between cell-associated MR with its sulphated sugar ligands in cells lining venous sinuses in human spleen. Venous sinusoidal cells have a phenotype intermediate between endothelial and haematopoietic cells and express low levels of CD31, thrombomodulin and the alpha chain of CD8. It is unclear if these cells provide any means of selectivity regarding the cellular and/or soluble blood components of host or pathogen origin that will be retained in the splenic parenchyma beyond the physical restriction determined by the size and or shape of the intercellular slits. The presence of MR and its ligands at this unique anatomical site suggests a role for this receptor in the interaction with mannosylated and/or sulphated blood components that could modify the adhesion properties of the endothelial cells and alter their filtration capacity.

\section{Acknowledgements}

We thank Dr Ron Jaffe for helpful discussions and Dr John Davies for his advice on immunohistochemistry. Research has been funded by the Medical Research Council, UK, The Edward Jenner Institute for Vaccine Research and The Edward Abraham Research Fund.

\section{References}

1 Taylor ME, Conary JT, Lennartz MR, et al. Primary structure of the mannose receptor contains multiple motifs resembling carbohydrate-recognition domains. J Biol Chem 1990;265:12156-12162.

2 Harris N, Super M, Rits M, et al. Characterization of the murine macrophage mannose receptor: demonstration that the downregulation of receptor expression mediated by interferon-gamma occurs at the level of transcription. Blood 1992;80:2363-2373.

3 Ezekowitz RA, Sastry K, Bailly P, et al. Molecular characterization of the human macrophage mannose receptor: demonstration of multiple carbohydrate recognition-like domains and phagocytosis of yeasts in Cos-1 cells. J Exp Med 1990;172:1785-1794.

4 Liu Y, Chirino AJ, Misulovin Z, et al. Crystal structure of the cysteine-rich domain of mannose receptor complexed with a sulfated carbohydrate ligand. J Exp Med 2000;191:1105-1116.

5 Pontow SE, Kery V, Stahl PD. Mannose receptor. Int Rev Cytol 1992;137B:221-244.

6 Schweizer A, Stahl PD, Rohrer J. A di-aromatic motif in the cytosolic tail of the mannose receptor mediates endosomal sorting. J Biol Chem 2000;275:2969429700.

7 Leteux C, Chai W, Loveless RW, et al. The cysteine-rich domain of the macrophage mannose receptor is a multispecific lectin that recognizes chondroitin sulfates $\mathrm{A}$ and $\mathrm{B}$ and sulfated oligosaccharides of blood group Lewis(a) and Lewis(x) types in addition to the sulfated $N$-glycans of lutropin. J Exp Med 2000;191: 1117-1126.

8 Fiete DJ, Beranek MC, Baenziger JU. A cysteine-rich domain of the 'mannose' receptor mediates GalNAc-4SO4 binding. Proc Natl Acad Sci USA 1998;95:20892093.

9 Fiete D, Beranek MC, Baenziger JU. The macrophage/ endothelial cell mannose receptor cDNA encodes a protein that binds oligosaccharides terminating with SO4-4-GalNAcbeta1,4GlcNAcbeta or Man at independent sites. Proc Natl Acad Sci USA 1997;94:1125611261.

10 Taylor ME, Drickamer K. Structural requirements for high affinity binding of complex ligands by the macrophage mannose receptor. J Biol Chem 1993;268: 399-404.

11 Linehan SA, Martinez-Pomares L, Stahl PD, et al. Mannose receptor and its putative ligands in normal murine lymphoid and nonlymphoid organs: in situ expression of mannose receptor by selected macrophages, endothelial cells, perivascular microglia, and mesangial cells, but not dendritic cells. J Exp Med 1999;189:1961-1972.

12 Engering AJ, Cella M, Fluitsma D, et al. The mannose receptor functions as a high capacity and broad specificity antigen receptor in human dendritic cells. Eur J Immunol 1997;27:2417-2425.

13 Sallusto F, Cella M, Danieli C, et al. Dendritic cells use macropinocytosis and the mannose receptor to concentrate macromolecules in the major histocompatibility complex class II compartment: downregulation by cytokines and bacterial products. J Exp Med 1995;182:389-400.

14 Engering A, Geijtenbeek TB, van Vliet SJ, et al. The dendritic cell-specific adhesion receptor DC-SIGN internalizes antigen for presentation to $\mathrm{T}$ cells. J Immunol 2002;168:2118-2126.

15 Kraal G. Cells in the marginal zone of the spleen. Int Rev Cytol 1992;132:31-74.

16 Steiniger B, Barth P. Microanatomy and function of the spleen. Adv Anat Embryol Cell Biol 2000;151:1-101.

17 Weiss L. The spleen. In: L Weiss (ed). Histology, Cell and Tissue Biology. Elsevier Science Publishing Co: New York, 1983, pp 544-568.

$18 \mathrm{Yu} \mathrm{P}$, Wang Y, Chin RK, et al. B cells control the migration of a subset of dendritic cells into $\mathrm{B}$ cell follicles via CXC chemokine ligand 13 in a lymphotoxin-dependent fashion. J Immunol 2002;168: 5117-5123.

19 Berney C, Herren S, Power CA, et al. A member of the dendritic cell family that enters $B$ cell follicles and stimulates primary antibody responses identified by a mannose receptor fusion protein. J Exp Med 1999; 190:851-860.

20 Martinez-Pomares L, Kosco-Vilbois M, Darley E, et al. FC chimeric protein containing the cysteine-rich domain of the murine mannose receptor binds to macrophages from splenic marginal zone and lymph node subcapsular sinus and to germinal centers. J Exp Med 1996;184:1927-1937.

21 Taylor PR, Zamze S, Stillion RJ, et al. Development of a specific system for targeting protein to metallophilic macrophages. Proc Natl Acad Sci USA 2004;101: 1963-1968.

22 Taylor PR, Gordon S, Martinez-Pomares L. The mannose receptor: linking homeostasis and immunity 
through sugar recognition. Trends Immunol 2005;26: 104-110.

23 Jordens R, Thompson A, Amons R, et al. Human dendritic cells shed a functional, soluble form of the mannose receptor. Int Immunol 1999;11:1775-1780.

24 Martinez-Pomares L, Mahoney JA, Kaposzta R, et al. A functional soluble form of the murine mannose receptor is produced by macrophages in vitro and is present in mouse serum. J Biol Chem 1998;273: 23376-23380.

25 Martinez-Pomares L, Reid DM, Brown GD, et al. Analysis of mannose receptor regulation by IL-4, IL10, and proteolytic processing using novel monoclonal antibodies. J Leukoc Biol 2003;73:604-613.

26 Martinez-Pomares L, Crocker PR, Da Silva R, et al. Cell-specific glycoforms of sialoadhesin and CD45 are counter-receptors for the cysteine-rich domain of the mannose receptor. J Biol Chem 1999;274:35211-35218.

27 Banerji S, Ni J, Wang SX, et al. LYVE-1, a new homologue of the CD44 glycoprotein, is a lymphspecific receptor for hyaluronan. J Cell Biol 1999;144: 789-801.

28 Morris L, Crocker PR, Hill M, et al. Developmental regulation of sialoadhesin (sheep erythrocyte recep- tor), a macrophage-cell interaction molecule expressed in lymphohemopoietic tissues. Dev Immunol 1992;2:7-17.

29 Harms G, Dijkstra CD, Hardonk MJ. Glycosyl receptors in macrophage subpopulations of rat spleen and lymph node A comparative study using neoglycoproteins and monoclonal antibodies ED1, ED2 and ED3. Cell Tissue Res 1990;262:35-40.

30 Irjala H, Johansson EL, Grenman R, et al. Mannose receptor is a novel ligand for L-selectin and mediates lymphocyte binding to lymphatic endothelium. J Exp Med 2001;194:1033-1042.

31 Maatta M, Liakka A, Salo S, et al. Differential expression of basement membrane components in lymphatic tissues. J Histochem Cytochem 2004;52: 1073-1081.

32 Mistry PK, Wraight EP, Cox TM. Therapeutic delivery of proteins to macrophages: implications for treatment of Gaucher's disease. Lancet 1996;348:15551559.

33 Boven LA, van Meurs M, Boot RG, et al. Gaucher cells demonstrate a distinct macrophage phenotype and resemble alternatively activated macrophages. Am J Clin Pathol 2004;122:359-369. 